further complicated by the finding that it can make disulphide bonds in reduced lysozyme in vitro ${ }^{12}$.

It will be of great interest to determine the mechanism that allows Erv2p to use molecular oxygen to make disulphide bonds, because no mechanism for such a reaction is known ${ }^{4}$. The array of proteins involved in disulphide bond metabolism is rich in such unique enzymes. For example, DsbB catalyses disulphide bond formation by using quinones for oxidizing equivalents ${ }^{6,7}$. DsbD performs transmembrane electron transfer by using cysteine residues, including some that are membrane-embedded ${ }^{8}$. Despite their important roles in cells, the molecular mechanisms of these enzymes are still poorly understood ${ }^{7,8}$.

Erv2p belongs to the Erv1p/ALR protein family $y^{4,12}$. Its members share domains of $\sim 100$ amino acids, with 30\% identity, that contain a CXXC motif, a feature of thioredoxin-like proteins. However, there are no other similarities to thioredoxins. Ervlp/ALR proteins are found in lower $\mathrm{r}^{4,13}$ and higher ${ }^{14}$ eukaryotes and in some virus$\mathrm{es}^{15}$. They are distributed in different subcellular compartments, including the $\mathrm{ER}^{4}$, cytoplasm $^{15}$, mitochondrial intermembrane space $^{13}$, and extracellular space ${ }^{14}$. Some are essential for the viability of cells ${ }^{13}$ or for the propagation of viruses ${ }^{15}$, indicating key roles in life. As proteins of this family exhibit oxygen-dependent thiol-oxidase activity in vitro ${ }^{13,14}$ similar to that of Erv2 $\mathrm{p}^{4,12}$, they are postulated to perform redox reactions in vivo, for example by catalysing disulphide bond formation like Erv2p or perfoming regulatory functions that involve protein thiols. In contrast to Erv2p, however, none of these proteins has been linked to a defined step in vivo. Adding to the confusion over the roles of such proteins is the knowledge that they can act either as oxidants or as reductants, depending on the redox state of their subcellular environment ${ }^{2}$.

The remarkable distribution of proteins of the Erv1p/ALR family in many cellular compartments raises the possibility that the formation of structural disulphide bonds might not be restricted at all times or under all conditions to the ER of eukaryotes or to the bacterial periplasm. Indeed, the E10R protein $^{15}$, a viral member of this family, was recently demonstrated to be required for disulphide bond formation in viral proteins in the cytoplasm. In addition, studies of redox pathways in the bacterial cytoplasm show that, with only one or two mutations, the cytoplasm can be converted to an environment in which the efficient formation of protein disulphide bonds takes place ${ }^{16}$. Further studies on the Erv1p/ALR family might expand the frontiers of the world of oxidative protein folding in cells. Hiroshi Kadokura and Jon Beckwith are at the Department of Microbiology and Molecular Genetics, Harvard Medical School, Boston,

Massachusetts 02115, USA

e-mail: jbeckwith@hms.harvard.edu

1. Anfinsen, C. B., Haber, E., Sela, M. \& White, F. H. Proc. Natl. Acad. Sci. USA 47, 1309-1314 (1961).

2. Ritz, D. \& Beckwith, J. Annu. Rev. Microbiol. 55, 21-48 (2001).

3. Frand, A. R., Cuozzo, J. W. \& Kaiser, C. A. Trends Cell Biol. $10,203-210(2000)$

4. Sevier, C. S., Cuozzo, J. W., Vala, A., Aslund, F. \& Kaiser, C. A. Nature Cell Biol. 3, 874-882 (2001)

5. Bardwell, J. C., McGovern, K. \& Beckwith, J. Cell 67, 581-589 (1991).

6. Bader, M., Muse, W., Ballou, D. P., Gassner, C. \& Bardwell, J. C. Cell 98, 217-227 (1999).

7. Kadokura, H., Bader, M., Tian, H., Bardwell, J. C. A. \& Beckwith, J. Proc. Natl. Acad. Sci. USA 97, 10884-10889 (2000)

8. Katzen, F. \& Beckwith, J. Cell 103, 769-779 (2000).

9. Cuozzo, J. W. \& Kaiser, C. A. Nature Cell Biol. 1, 130-135 (1999).

10. Tu, B. P., Ho-Schleyer, S. C., Travers, K. J. \& Weissman, J. S. Science 290, 1571-1574 (2000).

11. Norgaard, P. et al. J. Cell Biol. 152, 553-562 (2001).

12. Gerber, J., Muhlenhoff, U., Hofhaus, G., Lill, R. \& Lisowsky, T. J. Biol. Chem. 276, 23486-23491 (2001).

13. Lange, H. et al. EMBO Rep. 2, 715-720 (2001).

14. Hoober, K. L., Sheasley, S. L., Gilbert, H. F. \& Thorpe, C. J. Biol. Chem. 274, 22147-22150 (1999).

15. Senkevich, T. G., White, C. L., Koonin, E. V. \& Moss, B. Proc. Natl. Acad. Sci. USA 97, 12068-12073 (2000).

16. Stewart, E. J., Aslund, F. \& Beckwith, J. EMBO J. 17, 5543-5550 (1998).

\title{
A unisex contraceptive drug target
}

Fertilization requires calcium signalling at several levels. First, sperm cell motility and chemotaxis are regulated by calcium currents induced by the sperm cells' interaction with the outer layers of the egg. Second, penetration of the outer layers of the egg by the sperm cell requires the exocytosis of enzymes from the sperm cells, a process called the acrosome reaction, which is also calcium-dependent. Last, after fertilization, calcium transients in the egg are required for its activation.

David Clapham and his colleagues now identify a new calcium channel, CaSper, which is specifically expressed in the tail region of sperm cells (see picture; left, phase-contrast image; right, immunofluorescence staining for CaSper) and is required for their motility and for male fertility (Nature 413, 603-609, 2001). Inactivation of the corresponding gene leads to male sterility in mice that are otherwise healthy. Sperm cells from these mice fail an in vitro fertilization assay and their motility is markedly reduced. However, sperm-cell adherence to the egg is normal, and mutant sperm cells can fertilize eggs provided that their extracellular matrix is removed enzymatically. Because of the specificity of its expression pattern, CaSper is an ideal target for contraceptive drugs for both men and women.

So how does CaSper work? It bears structural homology to voltage-dependent potassium channels and sequence homology to voltage-dependent calcium channels. Clapham and colleagues
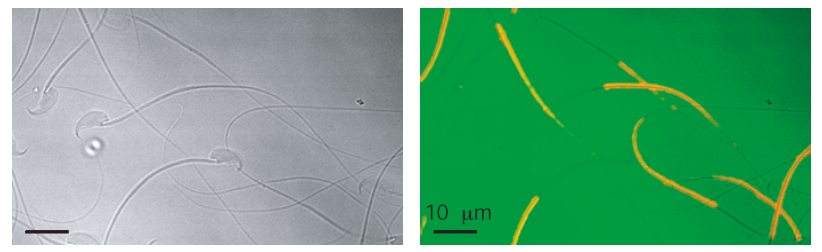

wished to study the electrophysiological properties of the CaSper channel, but patch clamp studies are still unsuccessful in the tail region of sperm cells because of technical limitations. The authors therefore turned to spermatocytes instead, but they saw no defects in voltage-gated calcium currents in CaSper-deficient cells. Experiments in which CaSper was ectopically expressed in Xenopus oocytes and in mammalian cells also proved inconclusive because no currents could be measured through CaSper by patch clamping. But CaSper-deficient sperm cells show no cyclicAMP-induced calcium influx, suggesting that CaSper is either gated by cyclic nucleotides or activated by the depolarization caused by native cyclic-nucleotide-gated channels. The authors speculate that CaSper-dependent channels could in turn activate calcium-dependent adenylyl cyclases and cAMP production in a positive feedback loop.

VALERIE FERRIER 